\title{
Fully textured monolithic perovskite/silicon tandem solar cells with $25.2 \%$ power conversion efficiency
}

Florent Sahli ${ }^{*}{ }_{\ddagger}{ }_{\ddagger}$ Jérémie Werner, ${ }_{\ddagger}{ }_{\ddagger}$ Brett A. Kamino, ${ }^{2}$ Matthias Bräuninger, ${ }^{l}$ Raphaël Monnard, ${ }^{1}$ Bertrand Paviet-Salomon, ${ }^{2}$ Loris Barraud, ${ }^{2}$ Laura Ding, ${ }^{2}$ Juan J. Diaz Leon, ${ }^{2}$ Davide Sacchetto, ${ }^{2}$ Gianluca Cattaneo, ${ }^{2}$ Matthieu Despeisse, ${ }^{2}$ Mathieu Boccard, ${ }^{1}$ Sylvain Nicolay, ${ }^{2}$ Quentin Jeangros ${ }^{1},{ }^{1}$ Bjoern Niesen, ${ }^{2}$ and Christophe Ballif ${ }^{1,2}$

${ }^{1}$ Ecole Polytechnique Fédérale de Lausanne (EPFL), Institute of Microengineering (IMT) Photovoltaics and Thin-Film Electronics Laboratory (PV-Lab), Rue de la Maladière 71b, 2002 Neuchâtel, Switzerland.

${ }^{2}$ CSEM, PV-Center, Jaquet-Droz 1, 2002 Neuchâtel, Switzerland.

E-mail: florent.sahli@epfl.ch, quentin.jeangros@epfl.ch

₹ These authors contributed equally to the manuscript

Keywords: organic-inorganic perovskite, multi-junction, silicon heterojunction, textured tandem, sequential deposition

\begin{abstract}
Tandem devices combining perovskite and silicon solar cells are promising candidates to achieve power conversion efficiencies $>30 \%$ at reasonable costs. State-of-the-art monolithic 2-terminal perovskite/silicon tandems have so far featured silicon bottom cells that are polished on their front side to be compatible with the perovskite fabrication process. This concession leads to higher potential production costs, higher reflection losses and non-ideal light trapping. To tackle this issue, we developed a top cell deposition process that achieves the conformal growth of multiple compounds with controlled optoelectronic properties
\end{abstract}


directly on the micron-sized pyramids of textured monocrystalline silicon. Tandem devices featuring a silicon heterojunction cell and a nanocrystalline silicon recombination junction demonstrate certified steady-state efficiencies of $25.2 \%$. Our optical design achieves a current density of $19.5 \mathrm{~mA} / \mathrm{cm}^{2}$ thanks to the silicon pyramidal texture and suggests a path for the realization of $30 \%$ monolithic perovskite/silicon tandems.

The photovoltaics market is dominated by the crystalline silicon (c-Si) technology with ever improving efficiencies and lowering costs. ${ }^{1}$ Thanks to continuous research, c-Si solar cells are approaching their theoretical Auger efficiency limit of $29.4 \%{ }^{2}$ with a current record at $26.7 \%{ }^{3,4}$ One approach to overcome this single-junction limit consists in stacking several absorbers of different bandgaps together in a multi-junction device to reduce thermalization losses. Recently, a power conversion efficiency of $32.8 \%$ was reached for a dual-junction combining III-V materials and $\mathrm{Si}^{5}{ }^{5}$ However, costly III-V deposition techniques have so far hindered the market penetration of this technology. Recently, organic-inorganic lead halide perovskite solar cells have been identified as a promising and potentially cheaper top cell alternative for tandems with bottom cells made of $\mathrm{c}-S i^{6}$ and chalcogenides. ${ }^{7}$ Perovskites exhibit a high power conversion efficiency (up to $22.7 \%$ at the single-junction level ${ }^{3,8}$ ), a sharp optical absorption edge ${ }^{9}$ and a tunable bandgap in the ideal range for silicon-based tandems (1.5-1.8 eV). ${ }^{10}$ These properties should theoretically allow perovskite/silicon tandem solar cells to reach efficiencies $>30 \%$ at reasonable production costs. ${ }^{6,11}$

Experimentally, values over $23 \%$ and $26 \%$ have been demonstrated for serially-connected 2terminal monolithic and mechanically-stacked 4-terminal configurations, respectively. ${ }^{12,13}$ So far, the latter approach has enabled higher performances, as both subcells are fabricated independently with fewer constraints imposed by the other cell (regarding texture, cell polarity and processing temperature) and because each subcell can be operated at its 
respective maximum power point. However, monolithic 2-terminal tandems are more attractive considering installation/system aspects and efficiency potential. ${ }^{6}$ They require fewer additional power electronic circuits (inverters and wires) and their high efficiency potential results from a gain in photocurrent due to reduced parasitic absorption (they do not require the two intermediate transparent conductive oxide electrodes, one for each subcell, needed in a 4terminal design). Despite these advantages, this approach comes with more constraints with respect to device processing. The main challenge is to develop a tailored top cell process that i) preserves the optoelectronic quality of the c-Si bottom cell and recombination layer, ii) ensures that the two subcells generate a high and equivalent photocurrent (at an open-circuit voltage as close as possible to the bandgap limit) and iii) yields conformal perovskite cells of high quality on double side textured c-Si cells to benefit increased light trapping in the infrared and from the reduced primary reflection due to double bounce effects (transforming a $10 \%$ reflection on a flat surface to $1 \%$ on a textured one as incident light reflected by a pyramid will likely reach an adjacent pyramid and be absorbed)

These first two challenges have already been addressed in state-of-the-art tandem devices. First, low-temperature $\left(<200^{\circ} \mathrm{C}\right)$ top cell deposition steps have been developed to prevent the degradation of silicon heterojunction (SHJ) bottom cells, ${ }^{14,15}$ a c-Si technology selected for its high open-circuit voltage ${ }^{16}$ and excellent near-infrared response. ${ }^{17}$ Secondly, the thickness of the perovskite absorber has been tuned in coordination with its bandgap, e.g. by depositing a 500 -nm-thick absorber consisting of a perovskite material with a bandgap of $1.63 \mathrm{eV}^{12}$ to achieve matched subcell currents $>18 \mathrm{~mA} / \mathrm{cm}^{2}$. The last challenge, the development of fully textured monolithic tandems directly compatible with monocrystalline Si industry standards, has so far not been mastered. Most perovskite deposition methods rely on solution-based spin coating. ${ }^{18}$ These methods do not produce conformal layers on fully textured monocrystalline Si bottom cells with their micron-sized (111) c-Si pyramids (see Figure 1). Due to this issue, current record devices are made with front side polished c-Si bottom cells. This leads to 
potentially higher production costs, ${ }^{6}$ strong front surface primary reflection losses and poor light trapping in the infrared part of the spectrum where c-Si absorbs weakly. The use of wafers without a front side texture limits the photocurrent to realistic values of $\sim 19 \mathrm{~mA} / \mathrm{cm}^{2}$ (including shadow losses induced by the front metal grid), a value that is $\sim 1 \mathrm{~mA} / \mathrm{cm}^{2}$ lower in comparison to a fully textured design. ${ }^{19,20}$ While various fabrication protocols based on physical and chemical vapor phase deposition have been developed, ${ }^{21-23}$ including hybrid sequential recipes combining thermal evaporation of the $\mathrm{Pb}$-containing precursor followed by spin coating of the organohalide compounds, ${ }^{7,24}$ perovskite cells deposited on textured c-Si bottom cells have not been reported yet.

To fully harvest the high photocurrent/efficiency potential of monolithic 2-terminal tandems, we developed a hybrid two-step deposition method combining sequential co-evaporation and spin coating ${ }^{25}$ to yield conformal perovskite absorber layers on textured SHJ bottom cells. The other layers of the perovskite cell are produced by means of thermal evaporation, atomic layer deposition and sputter deposition. A nanocrystalline silicon recombination junction is used between the two subcells, providing a high resilience to shunts. ${ }^{26,27}$ Combining these features, we demonstrate a fully textured monolithic perovskite/ SHJ tandem with a current density of $19.5 \mathrm{~mA} / \mathrm{cm}^{2}$ and certified steady-state power conversion efficiency of $25.2 \%$.

\section{Conformal perovskite layers and cells on textured c-Si}

Figure 1a schematically illustrates the fully textured monolithic perovskite/SHJ tandem cells developed here. A double side textured SHJ bottom cell with the n-type hydrogenated amorphous silicon layer, a-Si:H(n), facing sunwards was capped on its front side by either an indium tin oxide (ITO) or a nanocrystalline hydrogenated silicon (nc-Si:H) recombination junction: The former is a transparent conductive oxide (TCO) often used as recombination junction in perovskite/SHJ tandem devices ${ }^{12,14,15,28}$ or as front and rear electrodes in SHJs, ${ }^{29}$ 
while the nc-Si:H recombination junction has shown good optical and electrical properties in a previous study focusing on tandems using front side polished wafers. ${ }^{26}$

The perovskite cell was then deposited in the $p-i-n$ configuration. The use of textured SHJ bottom cells precludes solution processing for the perovskite absorber and selective layers. Indeed, attempts to spin-coat them resulted in the accumulation of the materials in the c-Si valleys, while the summits remained bare (Figure S1). This created shunts between the front side transparent conductive oxide and the SHJ bottom cell. An alternative hybrid deposition approach was developed to prevent this issue. A 2,2',7,7'-tetra(N,N-di-tolyl)amino-9,9-spirobifluorene (spiro-TTB) hole-selective layer was first deposited by thermal evaporation (Figure S2a-c). The perovskite was produced using a two-step process by co-evaporating a porous lead iodide $\left(\mathrm{PbI}_{2}\right)$ and cesium bromide $(\mathrm{CsBr})$ template (Figure $\mathrm{S} 2 \mathrm{~d}$ ) before spin-coating an organohalide solution (formamidinium iodide, FAI, and formamidinium bromide, FABr, Figure S2e). The organohalide solution infiltrates the pores of the $\mathrm{PbI}_{2} / \mathrm{CsBr}$ scaffold, while the rest of the solution is ejected off the substrate during spin coating. This effect prevents any accumulation of organohalides, e.g. in the pyramid valleys (Figure S2e). The $\mathrm{Cs}_{\mathrm{x}} \mathrm{FA}_{1-}$ ${ }_{\mathrm{x}} \mathrm{Pb}(\mathrm{I}, \mathrm{Br})_{3}$ absorber was then crystallized by annealing at $150^{\circ} \mathrm{C}$ in ambient air. ${ }^{25}$ The electron-selective layers, here a stack of $\mathrm{LiF}$ and $\mathrm{C}_{60}$, were subsequently deposited by thermal evaporation. A buffer layer of $\mathrm{SnO}_{2}$ was then deposited by atomic layer deposition and indium zinc oxide (IZO) was sputtered onto the stack, serving as the transparent front electrode. $^{30,31}$ The front metal grid was made of evaporated $\mathrm{Ag}$, while $\mathrm{MgF}_{2}$ acted as antireflective coating. Scanning electron microscopy (SEM) images shown in Figure S2 detail the different steps of this process. Overall, this top cell deposition process does not exceed $150{ }^{\circ} \mathrm{C}$, which is compatible with the use of hydrogenated silicon thin films for the bottom cell and recombination junction. ${ }^{32}$

This process yields a perovskite top cell that fully covers the micron-sized Si pyramids, as confirmed by the atomic force microscopy (AFM) 3D reconstructions and SEM images 
shown in Figure 1b-d and Figure S2f-g. Furthermore, cross sections of the perovskite top cell of a fully textured tandem device prepared by focused ion beam (FIB) and imaged by SEM demonstrate that the perovskite layer is dense and uniformly thick (Figure 1e and Figure S2hi). A scanning transmission electron microscopy (STEM) energy-dispersive X-ray spectroscopy (EDX) map of the perovskite top cell indicates that the inorganic elements of the perovskite, namely $\mathrm{Cs}, \mathrm{Pb}, \mathrm{Br}$ and I, interdiffuse completely during the annealing step (Figure 1f). Additional SEM, STEM and EDX data characterizing the perovskite top cell at various magnifications are shown in Figure S3. Furthermore, X-ray diffraction data indicates a full conversion of $\mathrm{PbI}_{2}$ to the perovskite phase (Figure $\mathrm{S} 4$ ). 
a)
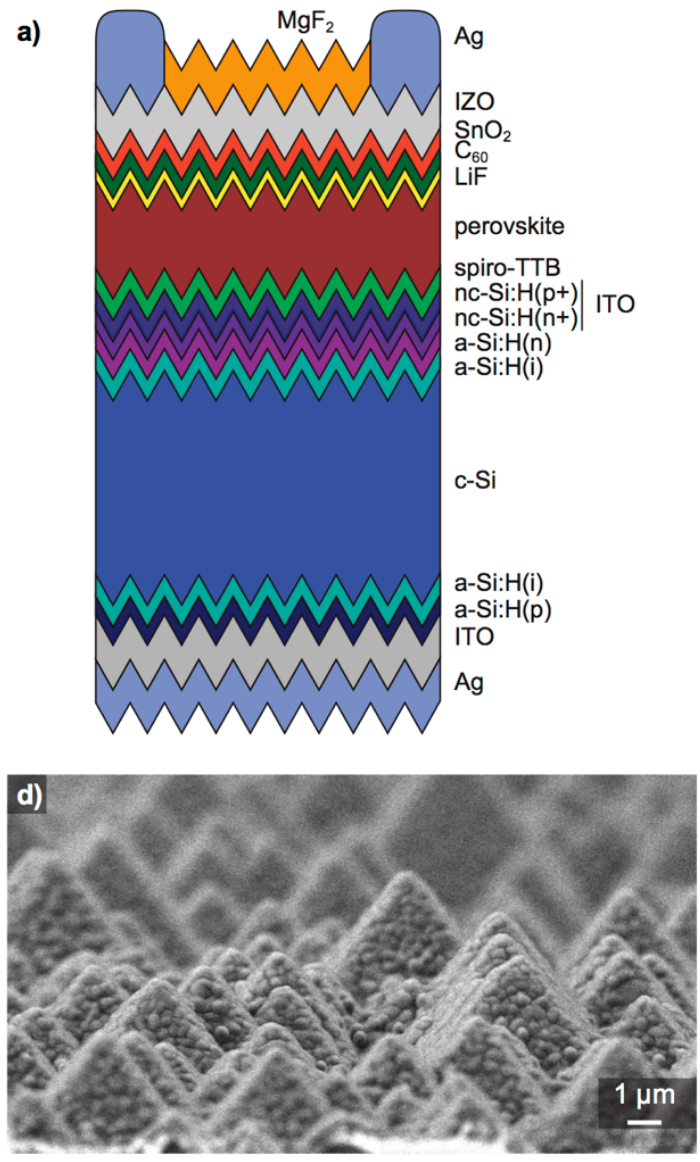

b)

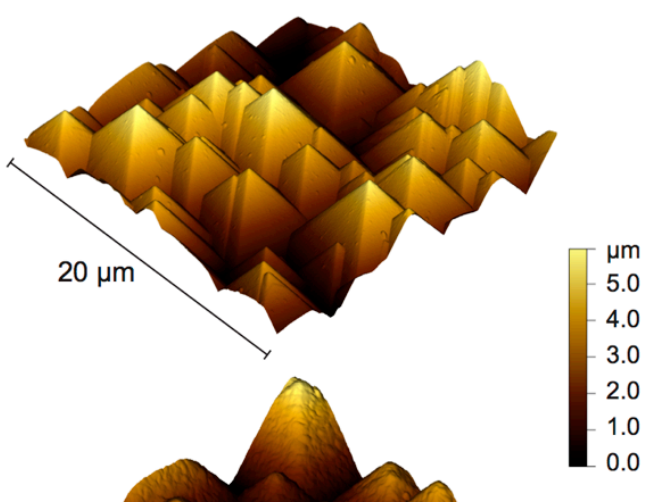

c)
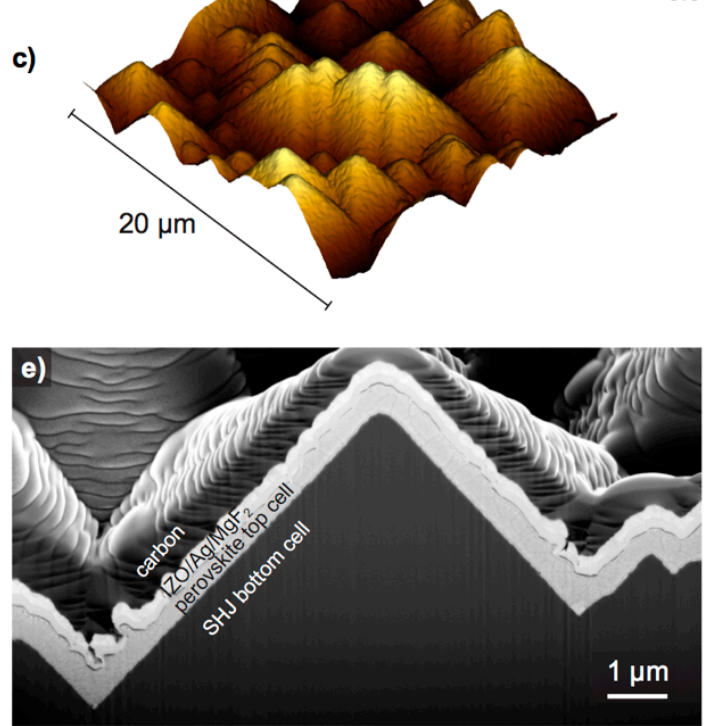

Net EDX intensity (a.u.)

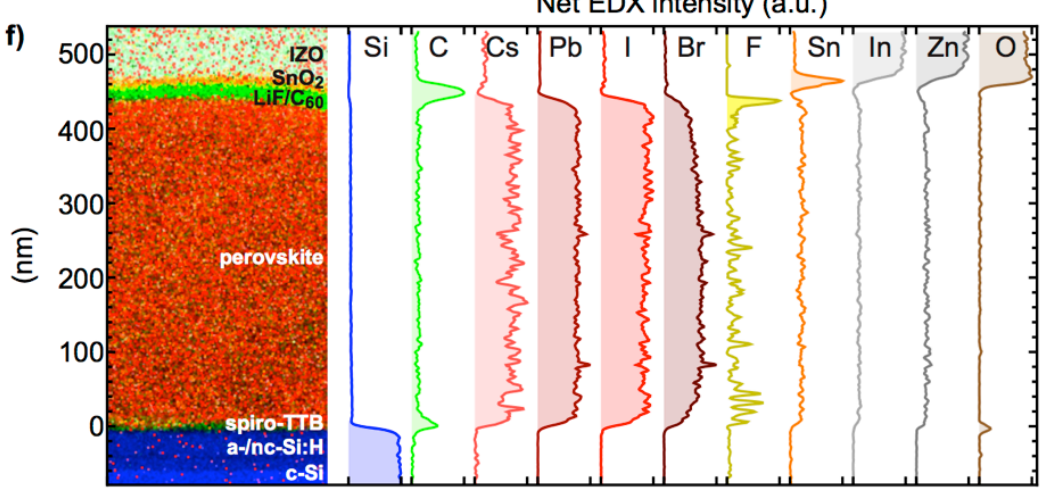

Figure 1: Cell design and microstructure of the perovskite top cell on textured silicon. a) Schematic view of a fully textured monolithic perovskite/SHJ tandem; AFM surface morphology 3D views of b) bare c-Si pyramids and c) c-Si pyramids covered with the perovskite layer; Secondary electron SEM image of d) the perovskite layer and e) a cross section of the full perovskite top cell deposited on the SHJ bottom cell, which was prepared at the position of a $\mathrm{Ag}$ finger by FIB (sample tilted to $54^{\circ}$, dimensions along the y axis to be multiplied by a factor 1.2 when compared to f). A carbon coating was applied to protect the top surface during the FIB milling process; f) Cross section STEM EDX map and corresponding line profiles of the relevant elements after background subtraction ( $\mathrm{K}$ 
ionization edges for all elements, with the exception of $\mathrm{Pb}$, for which the $\mathrm{L}$ edge is used). The noise in the F signal within the perovskite layer is an artefact of the background subtraction step.

\section{Benefits of nanocrystalline hydrogenated silicon}

The current density-voltage $(J-V)$ characteristics of the best tandem devices that combine the layers detailed above and either an ITO or an nc-Si:H junction are reported in Figure 2a. The $J-V$ curve of the ITO-based cell is comparable to that of a filtered single-junction SHJ with an open-circuit voltage $\left(V_{\mathrm{oc}}\right)<700 \mathrm{mV}$. This indicates that the top cell is shorted. On the other hand, the cell that employs an nc-Si:H layer stack as a recombination junction yields a $V_{\text {oc }}>$ $1780 \mathrm{mV}$ with a high efficiency (25.2\% at maximum power point, MPP, as discussed below).

a)

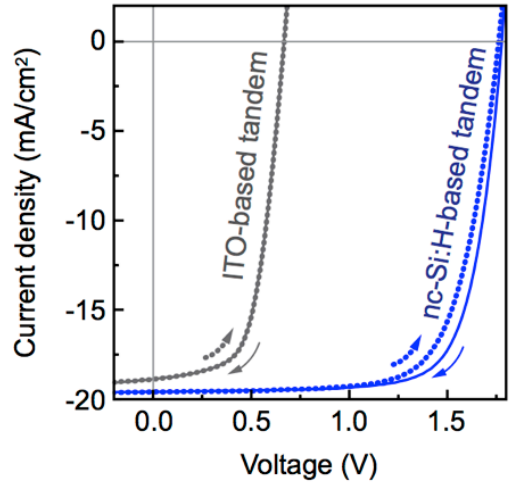

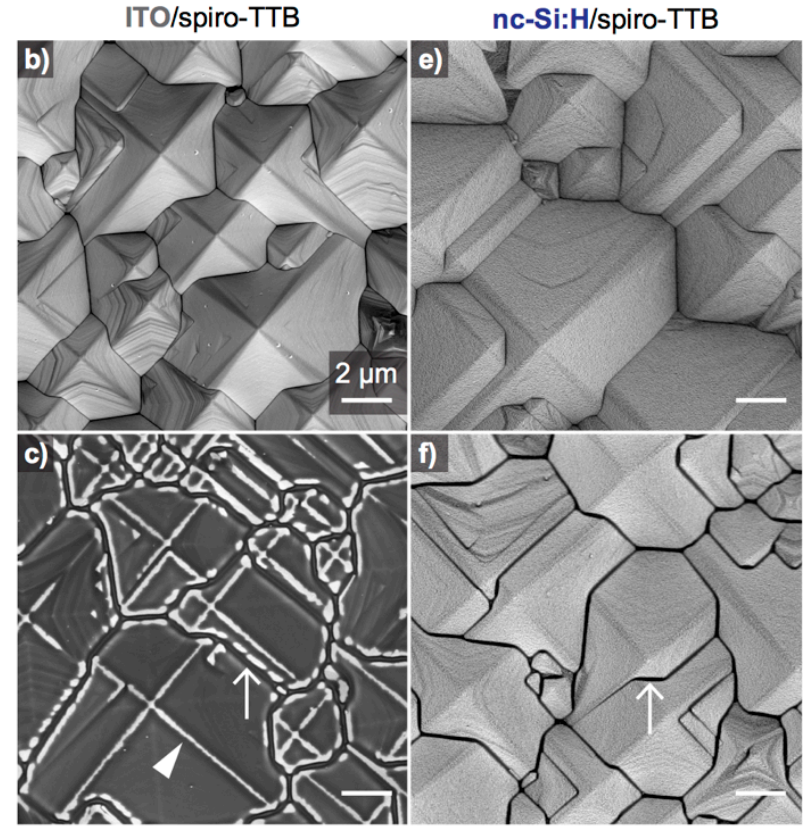

nc-Si:H/spiro-TTB/perovskite

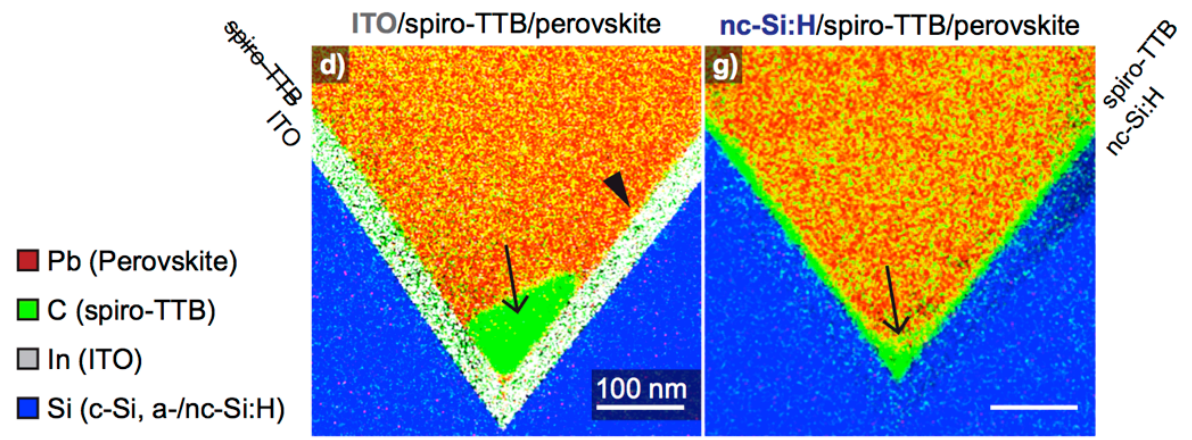

Figure 2: Comparison between different recombination junctions. a) $J-V$ characteristics of fully textured perovskite/SHJ tandems that feature either an ITO or an nc-Si:H recombination 
junction according to the architecture described in Figure 1. The cells were measured with a scan rate of $100 \mathrm{mV} / \mathrm{s}$; Top view secondary electron SEM images of spiro-TTB b) as deposited on the ITO recombination layer without any perovskite layer on top and c) after annealing at $150^{\circ} \mathrm{C}$ to simulate the effect of the temperature applied during the perovskite crystallization process; d) STEM EDX map of a cross section of the ITO/spiroTTB/perovskite stack at the bottom of a valley; SEM images of spiro-TTB e) as deposited on the nc-Si:H recombination junction without any perovskite layer on top and $\mathrm{f}$ ) after annealing at $150^{\circ} \mathrm{C}$; g) STEM EDX map of the nc-Si:H/spiro-TTB/perovskite stack at the bottom of a valley. Spiro-TTB slightly accumulates within the valleys during annealing when deposited on the nc-Si:H junction, while the effect is more severe with ITO (arrows, regions of dark contrast in the SEM images). This eventually leads to a local exposure of ITO (arrowheads, bright regions in the SEM images).

This significant difference in cell performance arises from a suboptimal ITO/spiro-TTB interface. While spiro-TTB is conformal after its evaporation on ITO (Figure 2b), it detaches from the pyramid summits and ridges during the $150^{\circ} \mathrm{C}$ annealing step required to crystallize the perovskite (Figure 2c). This locally uncovers the ITO, which appears as bright regions in the secondary electron SEM images shown in Figure 2c (indicated by arrowheads). The STEM EDX analysis shown in Figure 2d confirms the significant accumulation of spiro-TTB at the bottom of the Si valleys and its absence from some of the pyramid edges after annealing. These regions with a direct ITO/perovskite contact may act as recombination centers due to poor carrier selectivity. In comparison, when evaporated on nc-Si:H, spiro-TTB remains conformal, as confirmed by Figure 2e-g. Its thickness slightly increases at the bottom of the pyramid valleys after annealing (arrow in Figure $2 \mathrm{~g}$ ) but it still fully covers their edges. We assume that this effect is caused by a difference in surface tension between spiro-TTB and ITO when compared to nc-Si:H. Another reason that contributes to these differences in properties between ITO- and nc-Si:H-based tandem cells is the low lateral conductivity of nc$\mathrm{Si}: \mathrm{H}^{26,27,33} \mathrm{~A}$ high density of grain boundaries in the nc-Si:H recombination junction hinders lateral carrier transport, while the presence of large single grains that span across the junction improves transversal conductivity. ${ }^{27}$ This effect increases the shunt resistance and, e.g., 
enabled the production of larger-scale perovskite/front side polished SHJ tandems in a previous study. $^{26}$

\section{Improved optical system leading to a high efficiency}

After identifying the adequate combination of materials, the optical quality of our device architecture was assessed. External quantum efficiency (EQE) measurements highlight the advantage of switching from a polished front side to a double side textured architecture (Figure 3). While reflections occurring in the former design induce a current loss of $3.14 \mathrm{~mA} / \mathrm{cm}^{2}$ in the wavelength range $\lambda=360-1200 \mathrm{~nm}$, the introduction of a front texture reduces the overall reflectance, which drops to an equivalent integrated photocurrent value of $1.64 \mathrm{~mA} / \mathrm{cm}^{2}$. This optical gain stems directly from the presence of the c-Si front pyramidal texture, which i) suppresses the strong reflectance peak at $\lambda=850 \mathrm{~nm}$ (reflection at the front of the Si wafer), ii) lowers primary reflections at the front of the tandem cell (double bounce effect) and iii) increases light trapping of infrared light in the Si cell. Overall, the reflection losses in fully textured silicon monolithic tandem are below $2 \%$ in the range of $\lambda=350-$ $1000 \mathrm{~nm}$, excluding metallization.

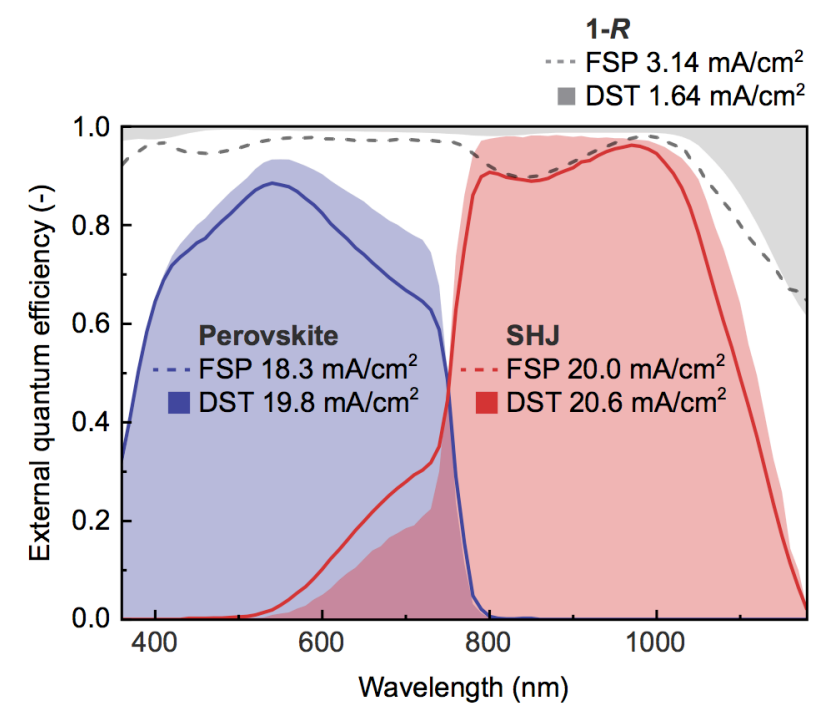

Figure 3: Improved optics with a fully textured architecture. EQE of perovskite/SHJ monolithic tandem cells with double side textured (DST) or front side polished (FSP) bottom 
cells, alongside corresponding total absorbance curves, 1-R, where $R$ is the reflectance of the device. These EQE and $1-R$ spectra do not include losses due to the front side metal grid.

A thickness/bandgap optimization to achieve current-matching conditions is shown in Figure S5. Overall, an optimum is reached for a 440-nm-thick perovskite absorber with a bandgap of $1.6 \mathrm{eV}$, with $20.1 \mathrm{~mA} / \mathrm{cm}^{2}$ generated in the perovskite and $20.3 \mathrm{~mA} / \mathrm{cm}^{2}$ in the SHJ (Figure $4 \mathrm{a}$, cross section shown in Figure 1f). The simple optical system presented here, which does not contain any light trapping feature other than the c-Si pyramids, exhibits a cumulative current of $40.4 \mathrm{~mA} / \mathrm{cm}^{2}$, a value that approaches that of record silicon devices $\left(42.5 \mathrm{~mA} / \mathrm{cm}^{2}\right)^{4}$ and surpasses what can realistically be achieved in front side polished devices without any complex light trapping scheme. ${ }^{11,19}$ This high EQE photocurrent translates to an improved device performance. Fully textured monolithic perovskite/SHJ tandems featuring a nc-Si:H recombination layer yield a $V_{\mathrm{oc}}>1.78 \mathrm{~V}$ (over an aperture area of $1.42 \mathrm{~cm}^{2}$, Figure $4 \mathrm{~b}$ ). This is comparable to the sum of the standalone subcells $\left(V_{\mathrm{oc}, \mathrm{SHJ}}\right.$ at $0.5 \mathrm{sun}=700 \mathrm{mV} ; V_{\text {oc, perovskite }}=$ $1050 \mathrm{mV}$, as shown in Figure S6). Thanks to the pyramidal Si texture, a short-circuit current density $\left(J_{\text {sc }}\right)$ of $19.5 \mathrm{~mA} / \mathrm{cm}^{2}$ is obtained, a value this time including shadow losses induced by the front metal grid (covering $2.3 \%$ of the aperture area). The highest efficiency $\eta$, certified by Fraunhofer ISE CalLab, is $25.52 \% \pm 0.70 \%$ obtained from the reverse scan and $24.09 \pm$ $0.66 \%$ from the forward scan (unencapsulated cell, Figure $4 b$ ). Other parameters retrieved from the $J-V$ reverse scan include a $V_{\mathrm{oc}}$ of $1788 \pm 12 \mathrm{mV}$, a $J_{\mathrm{sc}}$ of $19.53 \pm 0.37 \mathrm{~mA} / \mathrm{cm}^{2}$ and a fill factor FF of $73.0 \pm 1.45 \%$. Maximum power point tracking yields a certified steady-state value of $25.24 \pm 0.72 \%$ during $700 \mathrm{~s}$ (Figure S7). In-house measurement gave similar results (Figure S8). The $J-V$ hysteresis stems likely from the presence of mobile halide ions, as demonstrated in literature. ${ }^{34-37}$ For the same $J-V$ scan rate, this behavior is reduced when the temperature is increased (Figure S9) in accordance with literature data. ${ }^{38}$ Finally, the 
sequential perovskite deposition method presented here yields reproducible results (Figure S10).
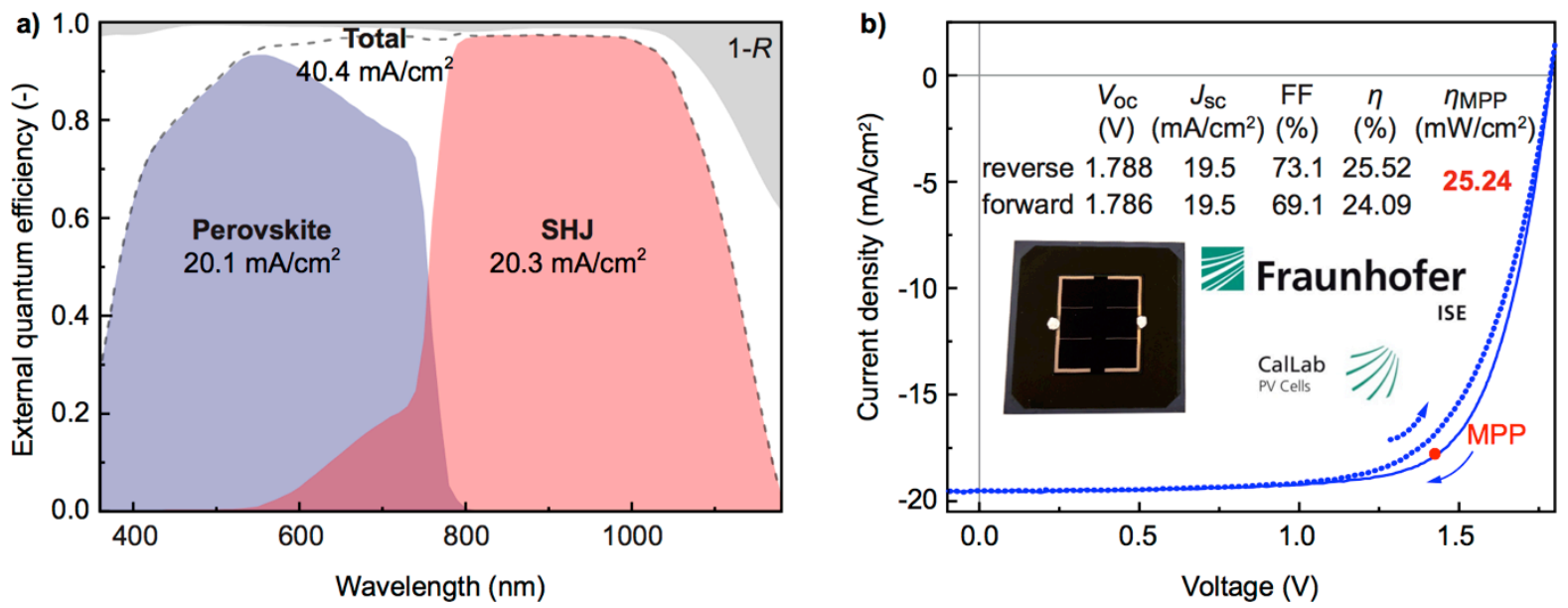

Figure 4: Certified performance of the fully textured perovskite/silicon tandem. a) EQE spectra of a current-matched fully textured monolithic perovskite/SHJ tandem cell featuring a $1.6 \mathrm{eV}$ perovskite absorber with a thickness of $440 \mathrm{~nm}$ alongside the $1-R$ curve, both excluding losses due to the front side metal grid; b) Corresponding certified $J-V$ data (1.42$\mathrm{cm}^{2}$ aperture area, device shown in the inset), which was measured with a scan rate of 100 $\mathrm{mV} / \mathrm{s}$

The perovskite composition employed in high efficiency tandem devices yields a lower performance at the single-junction level when compared to that inferred from tandem results due to several factors (Figure S6). Perovskite single-junctions feature an ITO/spiro-TTB interface (Figure S6), which is not optimal as demonstrated in Figure 2. Even if TEM indicates that spiro-TTB remains conformal on a flat ITO substrate (Figure S11), some dewetting might occur at the position of surface irregularities. In addition, the lateral conductivity of ITO reduces the shunt resistance of the device. These effects impact the FF and $V_{\mathrm{oc}}$ of perovskite single-junctions when compared to their top cell counterparts. Furthermore, with the deposition process used here, perovskite compositions that achieve current-matched conditions in tandems exhibit an increased surface roughness on flat surfaces. ${ }^{25}$ Opening the bandgap to $1.65 \mathrm{eV}$ yields a smoother film morphology and enables single-junction efficiencies of $15 \%$ at MPP, as reported in Ref. ${ }^{25}$. These differences between 
tandems and single-junctions highlight the necessity to optimize each configuration individually.

Overall, the tandem cell presented in Figure $4 \mathrm{~b}$ outperforms our best monolithic perovskite/SHJ tandems that feature a polished front side by more than $3 \%$ in absolute ${ }^{26}$ and the best in-house-made single-junction both sides-contacted SHJ cell by more than $2 \%{ }_{a}{ }^{39}{ }^{39}$ In addition, this efficiency surpasses the current reported record for monolithic perovskite/silicon tandems by $>1.6 \%{ }_{a b s .}{ }^{12}$ The results presented here demonstrate that our device architecture is optically optimized. Still, parasitic absorption could be reduced further, e.g. in the front electrode stack by thinning the $\mathrm{C}_{60} / \mathrm{SnO}_{2} / \mathrm{TCO}$ stack, opening the bandgap of the TCO and/or lowering its free carrier concentration. ${ }^{25}$ Moreover, metallization-induced shadow losses could be mitigated further by using screen-printed Ag lines, which have a more favorable aspect ratio when compared to thermally evaporated $\mathrm{Ag}$. For the cell size of $\sim 1 \mathrm{~cm}^{2}$ presented here, the $200 \mu \mathrm{m}$ wide evaporated Ag fingers lead to shadow losses of $2.3 \%$, a value that could be decreased to $0.6 \%$ by replacing them with $50 \mu \mathrm{m}$ wide screen-printed fingers $\left(J_{\mathrm{sc}}\right.$ gain of $\sim 0.3 \mathrm{~mA} / \mathrm{cm}^{2}$ ). Overall, reducing both parasitic absorption and shadow losses should enable a $J_{\mathrm{sc}}>20 \mathrm{~mA} / \mathrm{cm}^{2}$ for this cell size. With further realistic improvements in the $V_{\text {oc }}$ of the perovskite top cell to $1.2 \mathrm{~V}$ (while keeping a similar bandgap) ${ }^{40}$ and reaching a fill factor value of $80 \%$ (a value close to the one of state-of-the-art perovskite/SHJ tandems with a front side polished wafer $)^{12}$ could lead to perovskite/SHJ tandem with an efficiency over $30 \%\left(V_{\text {oc }}\right.$ $\left.=1875 \mathrm{mV}, \mathrm{FF}=80 \%, J_{\mathrm{sc}}=20 \mathrm{~mA} / \mathrm{cm}^{2}, \eta=30.0 \%\right)$.

\section{Device stability}

The devices developed above exhibit high efficiencies thanks to improved optics, a first prerequisite before any commercialization of the technology can be envisaged. The second key requirement for commercialization concerns device reliability. Planar single-junction produced with the hybrid method presented here pass 1000-hours damp heat degradation tests 
at $85^{\circ} \mathrm{C}$ and a relative humidity of $85 \%$ (Figure $\mathrm{S} 12$ ). The stability of perovskite-based devices was then investigated further by exposing fully textured perovskite/silicon monolithic tandem cells to continuous light irradiation. So far, such light soaking tests have been reported only for single-junction perovskite devices. ${ }^{41,42}$ Figure 5a shows the electrical characterization of a fully textured perovskite/SHJ tandem cell at MPP conditions under a continuous AM1.5G illumination without any UV-blocking filter. This measurement was carried out in ambient air (with a relative humidity level of $20-30 \%$ ) without encapsulation for 61 hours. $J-V$ scans were recorded regularly to monitor the evolution of the electrical parameters. After an initial light soaking period, the performance increases ${ }^{43}$ before remaining stable at $24 \%$ at MPP for 6 hours. The efficiency then starts to decrease linearly to $\sim 22 \%$ after 61 hours (linear rate of $0.15 \%$ rel $/$ hour). The corresponding $J-V$ data indicates that $J-V$ hysteresis increases during aging (Figure 5a-b). This behavior suggests that more ions become available for migration with time. This effect might be triggered by halide migration into the carrier-selective layers under bias, ${ }^{36,38}$ which leaves additional vacancies in the perovskite layer, or increased ionic conductivity from the diffusion of water into the hybrid perovskite material. ${ }^{44}$ Similar degradation tests were carried out using tandem cells that were encapsulated using cover glass sheets and a butyl-edge sealant (without any laminated encapsulant, Figure 5c). This simple encapsulation scheme reduces the degradation rate such that the cell retained $90 \%$ of its initial power conversion output after 270 hours under constant illumination at MPP. Similar to previously reported degradation data for single-junction perovskite cells, ${ }^{42}$ an exponential decay was also observed during the first 20 hours of illumination, followed by a linear regime for the next 250 hours (linear rate of $-0.03 \%$ rel $/$ hour). Both $J_{\text {sc }}$ and $V_{\text {oc }}$ decrease accordingly after an initial increase in $V_{\text {oc }}$ during the first few hours of operation (Figure $5 \mathrm{~d}$ ). Overall, the tandems presented here exhibit an encouraging light soaking stability when compared to single-junction literature data, ${ }^{42,45}$ with tandems retaining most of their electrical properties for tens (unencapsulated) to hundreds (encapsulated) of hours without any sudden failure. But 
further stability improvements are required to yield fully reliable devices. These will likely arise from the optimization of the device architecture, e.g. by replacing organic carrierselective layers by inorganic materials and by introducing buffer layers to prevent the migration iodine and its reaction with the $\mathrm{Ag}$ electrode. ${ }^{42,46} \mathrm{Ag}$ oxidation was found to occur at the front and back Ag metallization, the latter indicating that some iodide transport may occur in the form of volatile species ${ }^{47,48}$ (Figure S13). This halide vapor transport could be mitigated further by introducing a polymer encapsulant, a material that may also act as an UV light filter. $^{42}$

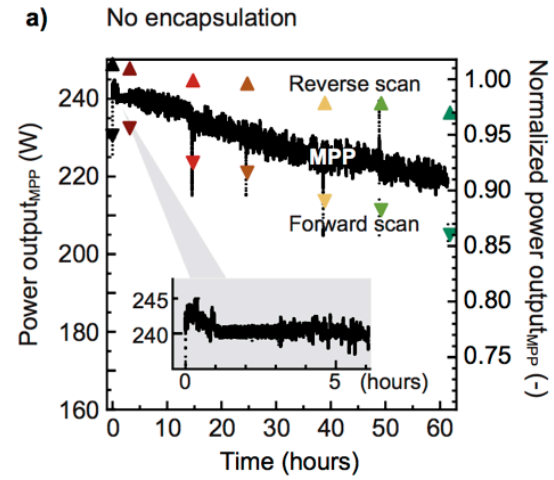

b)

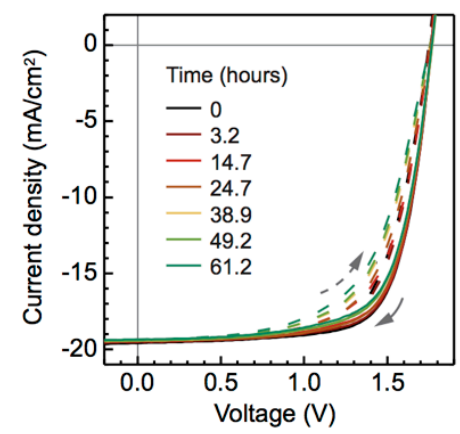

c) Glass/glass encapsulation with edge sealant

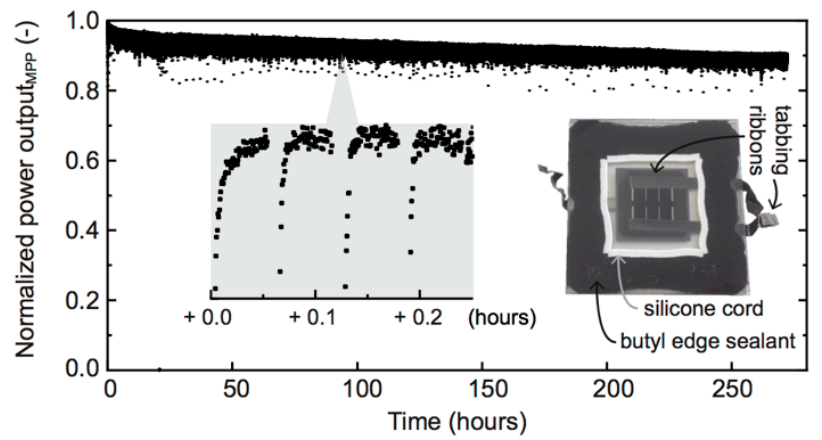

d)

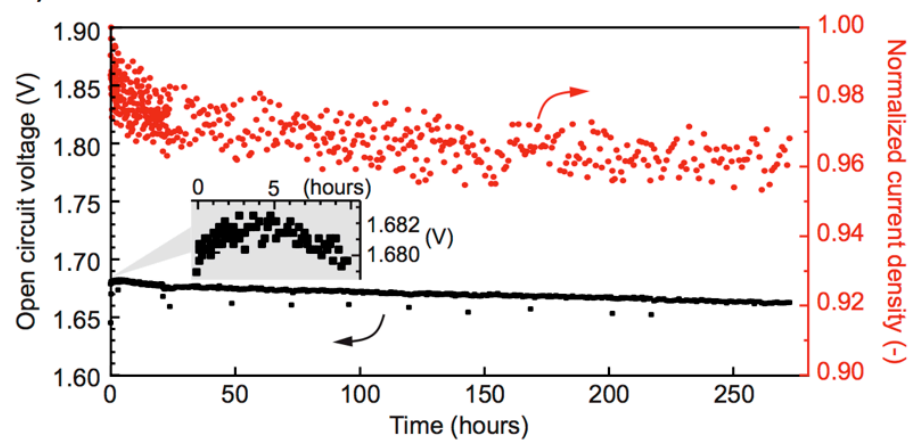

Figure 5: Device stability. a) MPP tracking of an unencapsulated fully textured perovskite/SHJ tandem solar cell measured at $25^{\circ} \mathrm{C}$ in air (relative humidity of $20-30 \%$ ); b) $J$ $V$ scans were recorded every 10 hours during the experiment shown in a). Power output values extracted from these reverse and forward $J-V$ scans are indicated in a) by triangles pointing upwards and downwards, respectively; c) MPP tracking of a glass/glass butyl-edge sealed fully textured perovskite/SHJ tandem solar cell in air (20-30\% relative humidity). This device had an initial efficiency of $23.8 \%$ before encapsulation (measured at MPP for $1000 \mathrm{~s}$ ) and $20.7 \%$ after encapsulation. This drop was mainly due to a decrease in $J_{\text {sc }}$ as no indexmatching encapsulant was present between the cell and the glass cover sheet. The spread in data points results from the recovery to MPP values after each $J-V$ scan (recorded every 5 min). The left inset illustrates this recovery behavior. The right inset is a picture of the encapsulated device; d) $V_{\text {oc }}$ and $J_{\text {sc }}$ values obtained from reverse $J-V$ scans. The inset shows 
the $V_{\text {oc }}$ during the first 10 hours of operation. Normalized power output efficiencies and currents are shown in c) and d) as the spectrum was not calibrated to 1 sun and no illumination mask was used on the encapsulated tandem cell. All $J-V$ measurements were recorded with a scan rate of $100 \mathrm{mV} / \mathrm{s}$.

\section{Outlook}

We demonstrate fully textured monolithic perovskite/silicon tandem solar cells and achieve a certified MPP efficiency of $25.2 \%$. Key to this result is a top cell fabrication process that does not require any modification of the textured c-Si bottom cell, therefore allowing the use of existing industrial textured monocrystalline $\mathrm{Si}$ wafers. The process yields conformal perovskite, carrier-selective and contact layers directly on the micron-sized c-Si pyramids. Electrical and microstructural characterization highlights the advantages of an nc-Si:H recombination junction over a conventional TCO, especially when featuring a thermally evaporated organic hole selective layer. Our design exploits the high photocurrent potential of monolithic tandems to achieve values of $19.5 \mathrm{~mA} / \mathrm{cm}^{2}$. The pyramidal texture of the c-Si bottom cell directly reduces primary reflection losses and enhances light trapping in the infrared. In addition, the stability of tandem devices to light soaking and electrical bias was monitored up to 270 hours.

Overall, the developed tandem fabrication procedure simplifies the overall process flow, yields high efficiencies thanks to improved optics and pave the way towards monolithic perovskite/silicon tandem cells with efficiencies $>30 \%$.

\section{Acknowledgements}

The authors thank Fabien Debrot and Christophe Allebé for SHJ wet-chemical processing, Jonas Geissbühler for help regarding the Arduino microcontroller and circuit design, Guillaume Charitat for the bottom cell deposition and Arnaud Walter, Soo-Jin Moon, Terry Chien-Jen Yang, Peter Fiala, Fan Fu for help regarding perovskite top cell processes and fruitful discussions. This work was funded by the Nano-Tera.ch Synergy project, the Swiss 
Federal Office of Energy under Grant SI/501072-01, the Swiss National Science Foundation via the Sinergia Episode (CRSII5_171000) and NRP70 Energy Turnaround PV2050 (407040) projects and the European Union's Horizon 2020 research and innovation program under Grant Agreement No. 653296 (CHEOPS).

\section{Author contributions}

F.S. and J.W. contributed equally to this work. F.S and J.W designed the experiments and carried out the devices fabrication. B.A.K. and M.Br. contributed to the development of the perovskite top cell. R.M., B.P-S., L.B., M.Bo. and M.D. developed and fabricated the silicon heterojunction bottom cells. D.S., L.D. and J.J.D.L developed the ALD buffer layer. G.C. and B.N. carried out the encapsulation for stability tests. Q.J. performed the FIB and TEM characterization and, with J.W., the SEM analysis. M.Br. recorded the AFM data. F.S., J.W. and Q.J. carried out data analysis and prepared the figures. J.W. carried out the degradation stability tests and analyzed the data. Q.J., B.N., M.Bo., M.D., S.N. and C.B. supervised different parts of the work. F.S. and Q.J. wrote the paper, all authors commented on the manuscript.

\section{References}

1. Battaglia, C., Cuevas, A. \& De Wolf, S. High-efficiency crystalline silicon solar cells: status and perspectives. Energy Environ. Sci. 9, 1552-1576 (2016).

2. Richter, A., Hermle, M. \& Glunz, S. . Crystalline Silicon Solar Cells Reassessment of the Limiting Efficiency for Crystalline Silicon Solar Cells. IEEE J. Photovoltaics 3, 1184-1191 (2013).

3. NREL Efficiency Chart. NREL Efficiency Chart. (2017).

4. Yoshikawa, K. et al. Exceeding conversion efficiency of $26 \%$ by heterojunction interdigitated back contact solar cell with thin film Si technology. Sol. Energy Mater. Sol. Cells 173, 37-42 (2017).

5. Essig, S. et al. Raising the one-sun conversion e ciency of III-V/Si solar cells to $32.8 \%$ for two junctions and 35.9\% for three junctions. Nat. Energy 17144, (2017).

6. Werner, J., Niesen, B. \& Ballif, C. Perovskite/Silicon Tandem Solar Cells: Marriage of Convenience or True Love Story? - An Overview. Adv. Mater. Interfaces 5, 1700731 (2018). 
7. Fu, F. et al. High-efficiency inverted semi-transparent planar perovskite solar cells in substrate configuration. Nat. Energy 2, 1234-1237 (2016).

8. Yang, W. S. et al. Iodide management in formamidinium-lead-halide-based perovskite layers for efficient solar cells. Science (80-. ). 356, 1376-1379 (2017).

9. De Wolf, S. et al. Organometallic halide perovskites: Sharp optical absorption edge and its relation to photovoltaic performance. J. Phys. Chem. Lett. 5, 1035-1039 (2014).

10. Eperon, G. E. et al. Formamidinium lead trihalide: a broadly tunable perovskite for efficient planar heterojunction solar cells. Energy Environ. Sci. 7, 982 (2014).

11. Grant, D. T., Catchpole, K. R., Weber, K. J. \& White, T. P. Design guidelines for perovskite/silicon 2-terminal tandem solar cells: an optical study. Opt. Express 24, 1454 (2016).

12. Bush, K. A. et al. 23.6\%-efficient monolithic perovskite/silicon tandem solar cells with improved stability. Nat. Energy 2, 17009 (2017).

13. Ramírez Quiroz, C. O. et al. Balancing electrical and optical losses for efficient 4terminal Si-perovskite solar cells with solution processed percolation electrodes. $J$. Mater. Chem. A 6, 3583-3592 (2018).

14. Werner, J. et al. Efficient Near-Infrared-Transparent Perovskite Solar Cells Enabling Direct Comparison of 4-Terminal and Monolithic Perovskite/Silicon Tandem Cells. ACS Energy Lett. 1, 474-480 (2016).

15. Albrecht, S. et al. Monolithic perovskite/silicon-heterojunction tandem solar cells processed at low temperature. Energy Environ. Sci. 9, 81-88 (2016).

16. Taguchi, M., Terakawa, A., Maruyama, E. \& Tanaka, M. Obtaining a higher Voc in HIT cells. Prog. Photovoltaics Res. Appl. 13, 481-488 (2005).

17. Holman, Z. C., Descoeudres, A., De Wolf, S. \& Ballif, C. Record Infrared Internal Quantum Efficiency in Silicon Heterojunction Solar Cells With Dielectric/Metal Rear Reflectors. IEEE J. Photovoltaics 3, 1243-1249 (2013).

18. Burschka, J. et al. Sequential deposition as a route to high-performance perovskitesensitized solar cells. Nature 499, 316-9 (2013).

19. Santbergen, R. et al. Minimizing optical losses in monolithic perovskite/c-Si tandem solar cells with a flat top cell. Opt. Express 24, A1288 (2016).

20. Schneider, B. W. et al. Pyramidal surface textures for light trapping and antireflection in perovskite-on-silicon tandem solar cells. Opt. Express 22, A1422-A1430 (2014).

21. Longo, G. et al. Fully Vacuum-Processed Wide Band Gap Mixed-Halide Perovskite Solar Cells. ACS Energy Lett. 3, 214-219 (2018).

22. Ioakeimidis, A., Christodoulou, C., Lux-Steiner, M. \& Fostiropoulos, K. Effect of PbI2 deposition rate on two-step PVD/CVD all-vacuum prepared perovskite. J. Solid State Chem. 244, 20-24 (2016).

23. Leyden, M. R., Jiang, Y. \& Qi, Y. Chemical Vapor Deposition Grown Formamidinium Perovskite Solar Modules with High Steady State Power and Thermal Stability. $J$. Mater. Chem. A 4, (2016).

24. Werner, J. et al. Efficient Monolithic Perovskite/Silicon Tandem Solar Cell with Cell Area $>1 \mathrm{~cm}$ 2. J. Phys. Chem. Lett. 7, 161-166 (2016).

25. Werner, J. et al. Complex Refractive Indices of Cesium-formamidinium-based Mixed Halide Perovskites with Optical Bandgaps from 1.5 to $1.8 \mathrm{eV}$. ACS Energy Lett. 3, 742-747 (2018).

26. Sahli, F. et al. Improved Optics in Monolithic Perovskite/Silicon Tandem Solar Cells with a Nanocrystalline Silicon Recombination Junction. Adv. Energy Mater. 1701609, 1701609 (2017).

27. Tomasi, A. et al. Simple processing of back-contacted silicon heterojunction solar cells using selective-area crystalline growth. Nat. Energy 2, 17062 (2017).

28. Wu, Y. et al. Monolithic perovskite/silicon-homojunction tandem solar cell with over 
22\% efficiency. Energy Environ. Sci. 10, 2472-2479 (2017).

29. de Wolf, S., Descoeudres, A., Holman, Z. C. \& Ballif, C. High-efficiency Silicon Heterojunction Solar Cells: A Review. Green 2, 7-24 (2012).

30. Morales-Masis, M., Martin de Nicolas, S., Holovský, J., De Wolf, S. \& Ballif, C. LowTemperature High-Mobility Amorphous IZO for Silicon Heterojunction Solar Cells. IEEE J. Photovoltaics 1-8 (2015).

31. Werner, J. et al. Sputtered rear electrode with broadband transparency for perovskite solar cells. Sol. Energy Mater. Sol. Cells 141, 407-413 (2015).

32. De Wolf, S. \& Kondo, M. Nature of doped a-Si:H/c-Si interface recombination. J. Appl. Phys. 105, 1-6 (2009).

33. Buehlmann, P. et al. In situ silicon oxide based intermediate reflector for thin-film silicon micromorph solar cells. Appl. Phys. Lett. 91, 143505 (2007).

34. Unger, E. L. et al. Hysteresis and transient behavior in current-voltage measurements of hybrid-perovskite absorber solar cells. Energy Environ. Sci. 7, 3690-3698 (2014).

35. Eames, C. et al. Ionic transport in hybrid lead iodide perovskite solar cells. Nat. Commun. 6, 7497 (2015).

36. Jeangros, Q. et al. In situ TEM analysis of organic-inorganic metal-halide perovskite solar cells under electrical bias. Nano Lett. 16, 7013-7018 (2016).

37. Li, C. et al. Iodine Migration and its Effect on Hysteresis in Perovskite Solar Cells. Adv. Mater. 28, 2446-2454 (2016).

38. Levine, I. et al. Interface-dependent ion migration/accumulation controls hysteresis in MAPbI3 solar cells. J. Phys. Chem. C 120, 16399-16411 (2016).

39. Descoeudres, A. et al. Low-temperature processes for passivation and metallization of high-efficiency crystalline silicon solar cells. Sol. Energy (2018). doi:10.1016/j.solener.2018.01.074

40. Correa-Baena, J.-P. et al. Identifying and suppressing interfacial recombination to achieve high open-circuit voltage in perovskite solar cells. Energy Environ. Sci. 10, 1207-1212 (2017).

41. Bryant, D. et al. Light and oxygen induced degradation limits the operational stability of methylammonium lead triiodide perovskite solar cells. Energy Environ. Sci. 9, 1655-1660 (2016).

42. Domanski, K., Alharbi, E. A., Hagfeldt, A., Grätzel, M. \& Tress, W. Systematic investigation of the impact of operation conditions on the degradation behaviour of perovskite solar cells. Nat. Energy 3, 61-67 (2018).

43. Nie, W. et al. Light-activated photocurrent degradation and self-healing in perovskite solar cells. Nat. Commun. 7, 11574 (2016).

44. Müller, C. et al. Water Infiltration in Methylammonium Lead Iodide Perovskite: Fast and Inconspicuous. Chem. Mater. 27, 7835-7841 (2015).

45. Duong, T. et al. Rubidium Multication Perovskite with Optimized Bandgap for Perovskite-Silicon Tandem with over 26\% Efficiency. Adv. Energy Mater. 7, 1-11 (2017).

46. Kato, Y. et al. Silver Iodide Formation in Methyl Ammonium Lead Iodide Perovskite Solar Cells with Silver Top Electrodes. Adv. Mater. Interfaces 2, 2-7 (2015).

47. Alberti, A. et al. Similar Structural Dynamics for the Degradation of CH3NH3PbI3 in Air and in Vacuum. ChemPhysChem 16, 3064-3071 (2015).

48. Han, Y. et al. Degradation observations of encapsulated planar $\mathrm{CH} 3 \mathrm{NH} 3 \mathrm{PbI} 3$ perovskite solar cells at high temperatures and humidity. J. Mater. Chem. A 3, 81398147 (2015).

49. Ying-Tung Hsiao \& China-Hong Chen. Maximum power tracking for photovoltaic power system. in Conference Record of the 2002 IEEE Industry Applications Conference. 37th IAS Annual Meeting (Cat. No.02CH37344) 2, 1035-1040 (IEEE, 
2002).

\section{Methods}

\section{Monolithic tandem fabrication}

SHJ bottom cells were fabricated using n-type double side textured silicon float-zone wafers with a resistivity of $1-5 \Omega$ and a thickness of $260 \mu \mathrm{m}$. Intrinsic and doped hydrogenated amorphous silicon layers were deposited on both sides of the wafer in a plasma-enhanced chemical vapor deposition (PECVD) reactor to passivate the silicon surface and to create the carrier-selective contacts. The SHJ bottom cells were produced by depositing the n-type contact at the front and the p-type contact at the back. The back electrode consisted of a sputtered ITO/Ag stack. The front side n-type amorphous silicon layer was then capped by the recombination junction, which consisted of either an ITO layer $(50 \mathrm{~nm} ; 190 \Omega / \mathrm{sq})$ or an ntype/p-type nc-Si:H layer stack. The former was sputtered, while the latter was deposited in the same PECVD reactor as the amorphous silicon layers $\left(<200{ }^{\circ} \mathrm{C}\right) .{ }^{26,27}$ The processed 4inch wafers were then laser cut to $2.5 \times 2.5 \mathrm{~cm}$ substrates.

Perovskite solar cells were fabricated in the $p-i-n$ configuration and deposited directly onto the ITO or nc-Si:H recombination junctions as follows. First, $12 \mathrm{~nm}$ of 2,2',7,7'-tetra(N,N-ditolyl)amino-9,9-spiro-bifluorene (spiro-TTB, >99 \%, Lumtec) was thermally evaporated to form the hole transport layer in an home-made evaporation system (base pressure of $<2 \cdot 10^{-6}$ mbar, working pressure of $<3 \cdot 10^{-6} \mathrm{mbar}$, evaporation rate of $0.2 \AA / \mathrm{s}$ measured by a quartz crystal monitor, aluminum oxide crucible, power applied of about $40 \mathrm{~W}$, reached with a ramp up of $10 \mathrm{~W} / \mathrm{min}$, substrate holder at room temperature). The perovskite absorber was then deposited using a sequential two-step method. First, $\mathrm{PbI}_{2}(>99.99 \%$, beads from Alfa Aesar) was co-evaporated with $\mathrm{CsBr}(>99.99 \%$, Abcr) in a Lesker mini-Spectros system (base pressure of $<2 \cdot 10^{-6}$ mbar, working pressure of $<5 \cdot 10^{-6} \mathrm{mbar}$, evaporation rate of $1 \AA / \mathrm{s}$ for $\mathrm{PbI}_{2}$ and $0.1 \AA / \mathrm{s}$ for $\mathrm{CsBr}$ as-measured by quartz crystal monitors, aluminum oxide crucibles, 
evaporation temperatures of $\sim 325^{\circ} \mathrm{C}$ for $\mathrm{PbI}_{2}$, reached with a ramp up of $20^{\circ} \mathrm{C} / \mathrm{min}$, and of $\sim 455^{\circ} \mathrm{C}$ for $\mathrm{CsBr}$, reached with a ramp up of $20^{\circ} \mathrm{C} / \mathrm{min}$, substrate holder at $30^{\circ} \mathrm{C}$ ).

Subsequently, a mixture of formamidinium bromide and iodide (in 1 to 3 molar ratio, $0.513 \mathrm{M}$ in ethanol, Greatcell solar) was spin-coated onto this template layer. This step was performed using dynamic solution dispensing at a spin speed of $4000 \mathrm{rpm}$ during $30 \mathrm{~s}$ in an inert atmosphere. The layers were then annealed at $150^{\circ} \mathrm{C}$ for $30 \mathrm{~min}$ in ambient air to crystallize the perovskite absorber. An electron-selective stack of LiF (1 nm, 99.98\%, SigmaAldrich $) / \mathrm{C}_{60}(15 \mathrm{~nm},>99.95 \%$, NanoC) was then thermally evaporated in an home-made evaporation system without breaking the vacuum in between these two depositions (base pressure of $<2 \cdot 10^{-6} \mathrm{mbar}$, working pressure of $>3 \cdot 10^{-6} \mathrm{mbar}$, evaporation rate of $0.1 \AA / \mathrm{s}$ for LiF and $0.3 \AA / s$ for $\mathrm{C}_{60}$ as-measured by quartz crystal monitors, aluminum oxide crucibles, power applied to the sources of $\sim 180 \mathrm{~W}$ for $\mathrm{LiF}$, reached with a ramp up of $40 \mathrm{~W} / \mathrm{min}$, and $\sim 80 \mathrm{~W}$ for $\mathrm{C}_{60}$, reached with a ramp of $20 \mathrm{~W} / \mathrm{min}$, substrate holder at room temperature). A buffer layer of $10 \mathrm{~nm}$ of $\mathrm{SnO}_{2}$ was deposited by atomic layer deposition using an Oxford instrument system at $100^{\circ} \mathrm{C}$ using tetrakis(dimethylamino)tin and $\mathrm{H}_{2} \mathrm{O}$ as precursors. $110 \mathrm{~nm}$ of indium zinc oxide (IZO) was then sputtered in an home-built system using a 4-inch target $\left(90 \% \mathrm{In}_{2} \mathrm{O}_{3}+10 \% \mathrm{ZnO}\right)$ with an $\mathrm{RF}$ power of $70 \mathrm{~W}$ (sheet resistance of $27 \Omega /$ sq, which was measured using a 4-point probe HMS-5000 Hall setup in the Van der Pauw configuration).

$120 \mathrm{~nm}$ of Ag was thermally evaporated at a rate of $2 \AA / \mathrm{s}$ through a shadow mask to form the front metal grid. Finally, an antireflective coating of $\mathrm{MgF}_{2}$ (100 nm, > 99.99\%, Umicore) was thermally evaporated at a working pressure of $<1 \cdot 10^{-5}$ mbar (base pressure of $<1 \cdot 10^{-5}$ mbar) at a rate of $2 \AA /$ s as-measured by a quartz crystal monitor. During all layer depositions by thermal evaporation, the deposition rate was first stabilized to the targeted value before opening the substrate shutter. All thicknesses in this section are given for $\mathrm{KOH}$-textured crystalline silicon substrates unless stated otherwise. These are determined by dividing the thicknesses deposited on flat glass witness samples by 1.7. 
The final tandem cell active area, defined by the size of the IZO front electrode deposited through a shadow mask, was $1.81 \mathrm{~cm}^{2}$, while the aperture area defined by the inside part of the metal frame was $1.42 \mathrm{~cm}^{2}$.

\section{Device characterization:}

Total reflectance spectra were recorded using a UV-VIS-NIR spectrophotometer (PerkinElmer Lambda 950 equipped with an integrating sphere). In-house $J-V$ measurements were obtained on a temperature-controlled vacuum chuck at $25^{\circ} \mathrm{C}$, if not stated otherwise, using a two-lamp (halogen and xenon) class AAA WACOM sun simulator with an AM1.5G irradiance spectrum at $1000 \mathrm{~W} / \mathrm{m}^{2}$. Independently certified SHJ cells were used to calibrate our in-house solar simulator. Shadow masks were used to define the illuminated area (here $1.42 \mathrm{~cm}^{2}$, determined by CalLab, Fraunhofer ISE). The cells were measured with a scan rate of $100 \mathrm{mV} / \mathrm{s}$ (using an integration time of $0.1 \mathrm{~s}$ and a delay of $0.1 \mathrm{~s}$ for each data point, the voltage step was $0.02 \mathrm{~V}$ ). Three-point weight maximum power point measurements were performed using an in-house LabVIEW code. ${ }^{49} \mathrm{~J}-\mathrm{V}$ measurements were taken after the cell had undergone maximum power point tracking for at least $500 \mathrm{~s}$. This was done to ensure that the cell reached a steady-state efficiency before $J-V$ characterization. EQE spectra were measured with a custom-made spectral response setup where the samples are irradiated with chopped light at a frequency of $232 \mathrm{~Hz}$ and the response measured with a lock-in amplifier. For tandem cells, blue and red light biases were used to saturate the complementary subcell. To measure each subcell near short-circuit conditions, $0.7 \mathrm{~V}$ and $1 \mathrm{~V}$ bias voltages were applied to the cell when measuring the top and bottom cell, respectively. Both EQE and $J-V$ measurements were carried out in air without any encapsulation if not stated otherwise.

Secondary electron SEM images were acquired with acceleration voltages ranging from 1 to $5 \mathrm{kV}$ using either an Everhart-Thornley or an in-lens detector (JEOL JSM-7500TFE or Zeiss NVision 40 microscopes). Cross sections of the fully textured perovskite/SHJ tandem were 
prepared for SEM or TEM analysis using a focused ion beam workstation (Zeiss NVision 40). Thin TEM lamellae were quickly transferred in air $(<2 \mathrm{~min})$ to a transmission electron microscope, either a FEI Tecnai Osiris or a double Cs corrected FEI TITAN Themis. STEM imaging using a high-angle annular dark-field detector was combined with energy-dispersive X-ray spectroscopy at an acceleration voltage of $200 \mathrm{kV}$ and a probe current of either 100 or $200 \mathrm{pA}$. More details about the sample preparation, the TEM analysis and potential artefacts associated with the technique can be found in Ref. ${ }^{36}$ XRD measurements were carried out in an Empyrean diffractometer (Panalytical) equipped with a PIXcel-1D detector. The diffraction patterns were measured using a $\mathrm{Cu} \mathrm{K} \alpha$ radiation (wavelength of $1.54 \AA$ ).

\section{Stability tests}

The unencapsulated device was measured at $25^{\circ} \mathrm{C}$ in ambient air $(20-30 \% \mathrm{RH})$ using a twolamp (halogen and xenon) class AAA WACOM sun simulator with an AM1.5G irradiance spectrum at $1000 \mathrm{~W} / \mathrm{m}^{2}$. Maximum-power point tracking measurements were performed for 61 hours using an in-house LabVIEW code. $^{24} J-V$ measurements were recorded approximatively every 10 hours following the same procedure explained above. The cells were kept under illumination between MPP and $J-V$ measurements.

A tandem cell was encapsulated between two $6 \mathrm{~cm} \times 6 \mathrm{~cm} \times 2 \mathrm{~mm}$ glass cover sheets. At the edges, a black butyl rubber edge sealant was applied. To prevent any inflow of the butyl edge sealant at elevated temperature, a frame of white silicone cord was placed on the cell side of the sealant. Ribbons with a conductive adhesive were placed on the front and rear side of the tandem cell to contact it. The encapsulated cell was light-soaked at ambient atmosphere (20$30 \% \mathrm{RH}$ ) using a Solaronix degradation system at 0.7 sun. During this measurement, the tandem cell was placed on a metal carrier plate with vacuum holes, kept at a controlled temperature of $30^{\circ} \mathrm{C}$. The cell was kept at its MPP using an in-house-written algorithm uploaded to an Arduino microcontroller. $J-V$ reverse and forward scans were initially recorded 
every 5 minutes and, after 30 hours of light soaking, every 30 minutes. The cell was kept under constant illumination.

Data and code availability

The experimental data and maximum power point tracking codes (LabVIEW and Arduino microcontroller) that support the findings of this study are available from the corresponding authors upon reasonable request.

\section{References}

49. Ying-Tung Hsiao \& China-Hong Chen. Maximum power tracking for photovoltaic power system. in Conference Record of the 2002 IEEE Industry Applications

Conference. 37th IAS Annual Meeting (Cat. No.02CH37344) 2, 1035-1040 (IEEE, 2002). 